\title{
Video thoracoscopic treatment of pectus excavatum with the minimally invasive technique nuss
}

\begin{abstract}
Summary
Introduction: The pectus excavatum is a mmon malformation of the anterior wall of the thorax, characterized by a depression of the sternum and impaired sternal condro joints. In most cases, congenital in origin and evolution. Reaches its greatest deformity in adolescence. For many surgical treatment techniques involving extensive resections of costal cartilages and mobilization of the sternum were used. In 1998 Donald Nuss describes a technique where video thoracoscopic introduces a sternal retro metal bar, which is inserted through a small incision on each side of the chest wall, malformation running without resection of costal cartilages or section and / or and/or sternal mobilization.
\end{abstract}

Goals: To report our experience in two surgical centers, for pectus excavatum the treatment with the technique of video thoracoscopic Nuss. Work retrospective, descriptive.

Materials and methods: Work retrospective, descriptive. From December 2015 to December 2019 surgically treat pectus excavatum 72 patients using the technique video thoracoscopic Nuss.

Results: 63 males and 9 females. The age range at the time of surgery was 7 to 18 years (mean 15.7). Six patients older than 18 years were excluded from the sample. The average rate for Haller in this population was 4.1. All patients got a metal bar sternal retro video thoracoscopic under control, side stabilizers were placed in all patients (65 bilateral and 7 unilateral). Epidural catheter was placed for postoperative analgesic analgesia management in $100 \%$ of cases. The average operating time was $94 \mathrm{~min}$. (70-110 $\mathrm{min})$. Intra-operative difficulties: one bar too high had to reposition itself in the same surgical procedure, the mean average hospitalization was 9.7 days (5 -27 days). Post operative complications:The most common early complication was pneumothorax self-limiting in patients $11(15.2 \%)$. The most severe complication was purulent pneumonia with effusion and pleural drainage needed toilette video thoracoscopy $1(1.3 \%)$. Three patients $(4.1 \%)$ had granulomas wound. Late migration of the bar that was surgically corrected with good outcome. In $65(90 \%)$ patients, the results were very good and good; $5(6.9 \%)$ regular and $2(2.7 \%)$ bad due to the presence of asymmetry chest.

Conclusions: Thoracoscopic technique video Nuss is safe, effective and reproducible for the treatment of patients with pectus excavatum. Parietal approach a minimum and low morbidity. The aesthetic results are very satisfactory.

Keywords: pectus excavatum, nuss technique, thoracoscopy, minimally invasive surgery
Volume 8 Issue 2 - 2020

\author{
Sergio Polliotto \\ Consultant Specialist in Pediatric Surgery, Argentina
}

Correspondence: Sergio Polliotto, Consultant Specialist in Pediatric Surgery,Argentina, Email spolliotto@intramed.net

Received: February 29, 2020 | Published: April 14, 2020
Abbreviations: CT, computed tomography; PE, pectus excavatum; ECG, electrocardiogram

\section{Introduction}

Pectus excavatum called (PE), pectus excavatum, sunken or funnel is a malformation of the anterior wall of the thorax, characterized by a deep depression of the sternum and sternal joints condro lower. The incidence of the pectus excavatum is 1 in 700 to 1000 live births. It is more common in males at a ratio $4 / 1$. It is the most common deformity of the chest wall. It has a hereditary and congenital most cases component. A birth can be mild or absent and is evident with progressive growth, especially in the adolescent period.Spontaneous regression is rare.Physical activity does not reverse the process of localized deformity. It may be associated with other abnormalities of the musculoskeletal system such as scoliosis, Marfan syndrome and Ehlers Danlos syndrome. In most patients are asymptomatic. In more severe cases the symptoms are: compression thoracic organs, of cardiac axis deviation, respiratory distress, lung infections, exercise intolerance, chest pain.
Spiral CT determines the index obtained by dividing Haller transverse and anteroposterior of the thorax diameter. The first description was made in 1594 by Bauhinus. ${ }^{1}$ In 1911 Meyer ${ }^{2}$ resecting surgical correction publishes two sunken cartilages (2nd and 3rd) without achieving improved anomaly. Sauerbuch ${ }^{3}$ laid the foundation of surgical correction based on extensive resection of costal cartilages and mobilization of the sternum. Since then, many open surgical techniques described, being the most used Ravitch ${ }^{4}$ described in 1949. In 1998 Donald Nuss ${ }^{4-7}$ discloses a technique that introduces a sternal retro metal bar through a small incision lateral thoracic, correcting the malformation without resection of costal cartilage and sternal section. The objective of this presentation is to describe our experience with the Nuss technique for minimally invasive correction of pectus excavatum.

\section{Material and method}

From December 2015 to December 2019, 72 patients with surgically treat pectus excavatum diagnosis technique using thoracoscopic Nuss video.The gender distribution was: 63 were males 
and 9 females. The age range at the time of surgery was 7 to 18 years (mean 15.7).Patients were evaluated: Chest computed tomography (CT), with an index measuring Haller, respiratory functional study. Echocardiogram, cardiological assessment. It was explained in detail to parents and patients the significance of this disease and especially the evolutionary course of postoperative postoperative. All patients are actively involved in the surgical decision.Indication is made with surgical diagnosis pectus excavatum clinician associated with two or more of the following criteria:

a. Evidence of progression of thoracic deformity subjective symptoms added.

b. Index increased to 3.25 Haller.

c. Compression heart on chest CT.

d. Nutrition consultation mitral valve prolapse, conduction abnormalities, arrhythmias, hypertrophy of cardiac axis deviation.

e. Studies of lung function with obstructive or restrictive lung disease.

f. Recurrence after conventional surgery.

\section{Surgical technique}

Under general anesthesia anaesthesia epidural catheter is placed for management of post-operative pain.

Supine position with both arms abducted.Right lateral approach with $5 \mathrm{~mm}$ trocar which $30^{\circ}$ optics is placed. Pneumothorax low flow and a pressure of $5 \mathrm{mmHg}$. two right and left side approaches $2 \mathrm{~cm}$ are made for positioning and fixing the bar.Under thoracoscopic viewing video using an introducer, we perform blunt dissection of the sternal retro space separating the pericardium to achieve externalize the introducer into the contralateral side of the chest. The metal rod is inserted into the chest with convexity upwards and positioned ana and after the rotation is convex making which corrects the deformity of the wall of the chest. It is fixed with stabilizers and side spaces are closed. pneumothorax Pneumothorax caused by a tubular probe placed underwater evacuated. This probe is removed before the end of surgery.

\section{Results}

In two multipurpose surgical centers, we operate 72 patients. The gender distribution was 63 males and 9 females.

The age range at the time of surgery was 7 to 18 years (mean 15.7). The average rate for Haller in this population was 4.1.Morphology: 52 and 18 were symmetrical pectus asymmetric.

\section{Symptoms and pathologies}

a. Mitral valve prolapse $3(4.1 \%)$

b. Scoliosis $2(8.3 \%)$

c. Marfan syndrome $3(4.1 \%)$

d. Palpitation $11(15.2 \%)$

e. Arrhythmias $15(20.8 \%)$

f. Chest pain $8(11.1 \%)$

\section{g. Exertional dyspnea $32(44.4 \%)$}

h. Low self-esteem $65(90.2 \%)$

i. Do not show bare chest on the beach 70 (97.2\%)

In all cases, an epidural catheter was placed for postoperative analgesic analgesia management with an average duration of 3 days. In all patients, sternal retro metal bar, video thoracoscopic under control was implemented.

Lateral stabilizers were placed in all patients, 65 patients bilaterally $(90.2 \%)$. No patient required placing two bars. The average operating time was 94 minutes (range 70-110 min). Intra-operative difficulties: a rod was positioned too high not having a visually satisfactory correction and repositioned in the same surgical procedure.

\section{Early complications}

$11(15.2 \%)$ pneumothorax postoperative (15.2\%) no clinical impact and spontaneous resolution. No case was drained.

$4(5.5 \%)$ pleural effusions (5.5\%) diagnosed by ultrasound, which did not require treatment.1 (1.3\%) pericardial effusion $(1.3 \%)$ not required drainage. $2(2.7 \%)$ wound secretions $(2.7 \%)$ treated locally with good results. A(1.3\%) pneumonia (1.3\%) with pleural effusion purulent fibrin was treated with toilette and video thoracoscopic pleural drainage. The bar was not removed and the patient had a good evolution and was discharged on postoperative 27.

In a patient $(1.3 \%)$ the epidural catheter is accidentally left at 24 hours. of post-operative, making it difficult pain management.We did not have conversions.Postoperative complications distance: 3 (4.1\%) wound granulomas $(4.1 \%)$. ( 2 of which were treated with primary closure toilette Local and with good performance. One patient remained a granuloma to remove the time bar.One patient had a lateral migration of the bar required surgical correction under general anesthesia with good postoperative evolution. In 2 patients $(2.7 \%)$ to remove the bar, it was surrounded bone tissue formation neo costal erosion, being difficult to remove.The mean average hospitalization was 9.7 days (5-27 days). The average residence time of the sweep was 2.5 years (range 2 years to 2 years and 9 months). 22 patients $(30.5 \%)$ remain still with the bar. The average time for the surgical extraction procedure was $45 \mathrm{~min}(30-80 \mathrm{~min})$, and was outpatient management in all cases.

\section{Discussion}

The pectus excavatum is the most common malformation of the chest wall in pediatrics.

The incidence is 1 in 700 to 1000 live births. ${ }^{8}$ It is more common in males at a ratio $4 / 1$. Most patients are asymptomatic. In more severe cases may cause compression of the thoracic organs producing cardiac axis deviation and / or and/or breathing difficulties, respiratory infections, exercise intolerance and chest pain.

The etiology is unknown, but many studies describe a familial tendency inherited and in most cases congenital. ${ }^{89} \mathrm{~A}$ birth can be mild or nonexistent. It becomes evident with progressive growth, especially in the adolescent period.Spontaneous regression or partial improvement of this defect is rare.Physical activity does not reverse the process of localized deformity.It may be associated with other abnormalities of the musculoskeletal system such as scoliosis, Marfan syndrome and Ehlers Danlos syndroms. ${ }^{8-10}$ All patients are 
were studied: CT scan, pulmonary function tests and cardiovascular evaluation, ECG and echocardiogram. The mitral valve prolapse is common in these patients and usually have no changes after surgery. ${ }^{8-}$ ${ }^{10}$ Conventional test pulmonary function evaluation and may be maybe normal heart at rest, presenting only pathological alterations in patients evaluated during intense exercise. ${ }^{9}$ The decision for surgery is based on clinical diagnosis of pectus excavatum severe with two or more of the following criteria:

a) Index Haller in greater TAC 3.25 and evidence of heart compression therein.

b) Pulmonary function studies indicating a restrictive or obstructive airway disease.

c) Cardiac assessment revealed a prolapsed mitral valve, abnormal rhythm, conduction defect, hypertrophy or deviation from the axis.

d) Deformity progression of symptoms associated with subjective.

e) Recurrence after previous open surgery or minimally invasive repair.

f) Psychological problems related to pathology.

This technique it can be performed at any age as there is no interference with the nuclei of growth, but most authors recommend be performed between 14 and $18 \cdot{ }^{11-14}$ Some patients report series operated from 6 years, which means long growth to the body structure of the adolescent period during which increases the possibility of generating a relapse. ${ }^{11}$ In our series we observed in very young patients the bar tends to be small with the growth of the child, burrowing rib and generating tissue neo formation neoformation which hinders extraction. (Two patients in our series). Secondarily young children have greater difficulty understanding and accepting post-operative pain so we prefer not to indicate surgery before age 14 , depending on the physical and psychological development of the patient. We believe that the only condition for early intervention is a very severe cardiopulmonary disease with symptomatic compression. (One patient in our series). All patients are actively involved in the surgical decision and if the patient does not clearly express its willingness to surgery, the surgery is not performed.

The dwell time of the bar varies according to the series. ${ }^{11,12} \mathrm{We}$ left the bar at least two years and a maximum of three years. Physical activity begins the post operative postoperative month and three months is complete. At the beginning of our experience, we restrict contact sports. With the evolution learned that patients can perform physical activity without limitations including contact sports. In 65 $(90 \%)$ patients $(90 \%)$, the results were very good and good; $5(6.9 \%)$ regular and $2(2.7 \%)$ bad due to the presence of asymmetry chest.

\section{Conclusions}

The pectus excavatum has a great impact on aesthetics affect selfesteem and personality development. Typically, these children are shy, inhibited and withdrawn, refrain from participating in activities in which the chest is exposed, as athletics, swimming beach etc. occurs in.At the correct deformity, there is a marked improvement in selfesteem.
Video thoracoscopic technique for treating Nuss pectus has a low morbidity with good aesthetic results and long-term stable and since published in 1998, it is the technique of choice in centers for correcting the pectus excavatum.

\section{Acknowledgments}

None.

\section{Conflicts of interest}

Author declare that there is no conflict of interest.

\section{Funding}

None.

\section{References}

1. Observations Bauhinus patients. LIVER'll Obserr.

2. Meyer L. Zurchirurqishen treatment of breasts funnel-born bel. Med 1991;42:364

3. Sauerbruch F. Surgical removal of the congenital funnel breast. Deustsche Chir. 1937;234:760.

4. Ravitch MM. The operative treatment of pectus excavatum. Ann Surg. $1949 ; 129: 429-444$.

5. Nuss D, Kelly RE, Croitoru DP, et al. Repair of pectus Excavatum Pediatric Endosurgery \& Innovative Techniques. 1998;2:205-221.

6. Nuss D, Kelly RE, Croitoru DP, et al. A 10-Year Review of a Minimally Invasive Technique for the Correction of pectus excavatum. JP Surg. 1998;33:545-552.

7. Croitoru DP, RE Kelly, Goretsky MJ, et al. Experience and Modification Update for the Minimally Invasive pectus excavatum Nuss Technique for Repair in 303 Patiens. JP Surg. 2002;37:437-445.

8. Creswick H, Stacey M, R Kelly, et al. Family Study of the inheritance of pectus excavatum. JP Surg. 2006;41:1699-1703.

9. Kelly R, Quinn A, Varela P, et al. Dysmorphology deformities of the chest wall: frequency distribution of the subtypes of pectus excavatum typical and rare subtypes. Arch Bronconeumon. 2013;49 (5):196-200.

10. Hernandez S, Fernandez A, Ramirez M, et al. The correction of pectus excavatum complicated with the support of extracorporeal circulation. Cir Pediatr. 2011;24:44-47.

11. DonaldNuss, Robert E Kelly. Minimally invasive repair of the pectus excavatum. Cir Pediatr. 2002;15:1-2.

12. Binkovitz L, Zendejas BMoir C, et al. Nuss bar migrations: occurrence and classification. Pediatr Radiol. 2016:46(13):1797-1803.

13. Haller JA. Severe chest constriction from growth retardation wall after too extensive and too early pectus excavatum repair: An alert. Ann Thorac Surg. 1995;60:1857-1864.

14. Martinez D, J Juanne, Stein U, et al. The effect of costal cartilageon chest wall resection development. Pediatr Surg. 1990;5:170-173. 